\title{
Research on the Influencing Factors of Commercial Retail Location
}

\section{Chujiang Xue}

School of Economics, Shanghai University, Shanghai, China

\section{Email address:}

chujiangx@sina.com

\section{To cite this article:}

Chujiang Xue. Research on the Influencing Factors of Commercial Retail Location. Science Innovation. Vol. 5, No. 3, 2017 , pp. 175-179. doi: 10.11648/j.si.20170503.19

Received: March 29, 2017; Accepted: April 14, 2017; Published: April 20, 2017

\begin{abstract}
City development hinges on the business activities within a city. The space structure of business activities is always an essential subject in urban geography and urban economics researches. As the progress of economical growth, the relationship between customers and big shopping malls becomes closer and closer. There are conspicuous differences and distinct spatial agglomeration among different types of business sites location. To be succinct, land price, traffic accessibility and feature aggregation are all important factors influencing the location of different business sites in diverse extent.This paper is aiming to analyze factors that influence the location of business sites and circles using analytic hierarchy process, in order to provide some reference significance for related issues.
\end{abstract}

Keywords: Commercial Retail, Analytical Hierarchy Proess, Yaahp, Contributing Factors, Area Characteristic, Locating

\section{商业零售业选址影响因素研究}

\section{薛楚江}

经济学院, 上海大学, 上海市, 中国

\section{邮箱}

chujiangx@sina.com

摘要: 城市的发展与城市中的商业活动密切相关, 城市中商业活动的空间结构一直是城市地理学和经济学所研究的重 点领域。随着经济的发展, 商业活动中与人们日常生活联系比较紧密的有大型购物商场、超市等, 各种类型网点空间 分布差异性较大，而且其空间集聚表现也各不相同。地价、交通及集聚特征等都是影响商业网点分布的因素，不同因 素对不同商业网点分布的影响具有较大的差异。本文就是以商业零售业选址的影响因素为例运用层次分析法来分析商 业活动以及商圈建设选址的影响因素。解决了如何运用数学方法分析处理商业零售业选址影响因素的模糊问题, 为商 业零售业的选址提供有益的参考。

关键词：商业零售业, 层次分析法, yaahp软件, 影响因素, 区位特征, 选址

\section{1. 引言}

中国经济发展已进入新常态, 商业零售业也进入了精 细化管理的轨道。近年来, 连锁超市等商业零售业的空间
分布和区位选择成为了学术研究的热点。零售业是企业战 略选择中灵活性最差的, 但是零售情况的好坏直接影响企 业的营收, 而且还会显著影响企业今后的网点布局以及后 期的扩张, 从而影响后期的战略运行及战略选择 [1]。通 
过对国内外成功商业零售企业成功的原因进行分析, 其中 企业获得成功的一个共同点是选址。企业的选址通常是企 业家根据选址经验甚至是主观意愿的方法进行选择, 缺乏 必要的数据定量分析, 通过研究发现国内商业零售业的选 址成功率一直较低, 缺乏成功选址的经验 [2]。本文就是 运用层次分析法从地理因素、人口因素和竞争因素等三个 角度来分析商业活动以及商圈建设选址的影响因素, 从而 得到综合分析选址的评价模型, 为商业零售业的精细化管 理和选址操作提供有益的参考。

\section{2. 国内外研究成果}

\section{1. 国外研究成果}

国外学者从十九世纪末二十世纪初开始对商业零售 业区位选择进行研究, 共经历了四个阶段：20世纪20-30 年代的商业零售业空间竞争与市场区位研究阶段； $30-60$ 年代以中心地理论为指导的新古典宏观区位研究阶段; 60-80年代的以消费者行为理论为指导的零售商业空间结 构研究阶段; 80 年代至 21 世纪初以社会空间因素和行为感 知为重点的商业零售业区位研究阶段 [3]。近十年的研究 基本集中在商品流转、零售业国际化、零售中心吸引力、 市场网络、商圈与集聚经济、购物中心的吸引、商圈扩张 与竞争、商圈与经济集聚、商业空间重构与消费者选择行 为研究等方面, 由此可见外国学者对商业区位选择的研究 已经颇为成熟。

\section{2. 国内研究成果}

中国对于区位选择理论的研究始于改革开放后期。在 1980到1990年间, 中国处于商业地理学及城市商业区位为 主的宏观研究阶段, 研究对象主要为商业地理学和城市商 业区位。商业地理学的发展、研究对象及性质和大城市商 业中心体系、演变趋势, 商业网点的类型及区位选择等问 题为这一时期学术界关注的焦点。20世纪 90 年代以后, 商 业区位理论的研究从宏观层面延伸到中观层面, 商业网点 的等级体系、空间格局、演变趋势以及结合中心地理论的 实证分析逐渐演变成为这一时期研究的重点 [4]。进入 21 世纪以来, 我国城市商业区位的研究转由中观层面向微观 层面的零售业百货商店区位、便利店及连锁店区位的研究 延伸，并通过GIS技术手段和空间计量模型等全新方法， 推动了城市商业零售业区位研究的发展。

\section{3. 研究方法}

层次分析模型 (analytichierarchyprocess, 简写AHP) 是美国运筹学家Satty教授于二十世纪70年代末提出的一 种实用的多方案或多目标的决策方法。这种方法适用于结 构较为复杂、决策准则较多而且不易量化的决策问题。其 思路简单明了, 合理地将定性与定量的方法结合起来, 对 决策者的推理过程层次化、数量化, 避免了决策者在结构 复杂和方案较多时逻辑推理上的失误 [5]。
层次分析法是一种将定量与定性方法有效结合的多 目标规划方法, 也是一种技术优化方法, 经过长时间的发 展现在已经成为一种成熟的研究方法, 如今已经在很多领 域有了广泛的应用。具体操作步骤 [6]如下所示:

第1步：明确问题，提出总目标。

第2步：根据各目标层建立层次结构图, 把问题分解 成若干个层次。第一层为总目标层; 第二层为中间层, 可 根据问题的性质分解成部门层、目标层 (准则层) 、约束 层等不同的层次; 最底层一般为措施层或方案层。

第3步: 求同一层上的权系数 (从高层到低层依次进 行）。假设当前层次上的因素为 $\mathrm{A}_{1}, \cdots, \mathrm{A}_{n}$, 相关的上一 层因素为 C (可以不止一个)。对每个层次元素两两逐次 进行比较, 按照规定的标度进行量化后写成矩阵的形式, 构造出判断矩阵。再根据两两比较的结果算出各因素最总 目标层的权重, 根据综合权重按最大权重原则确定最优方 案。设两两判别矩阵为 $\mathrm{a}_{i j}$, 则有: $\mathrm{a}_{i j}>0 ; \mathrm{a}_{i j}=1 / \mathrm{a}_{j i}(\mathrm{ij}=1$, $2, \cdots, n) ; a_{i j}=1(i=j$ 在总目标层 $(A)$ 下, 根据各相关因素 构造该级别的判别矩阵 $(A-B)$ 。这里需要引用 $1 \sim 9$ 标度对 重要性程度的判断结果进行量化, 标度表如表1所示。构 造 $(\mathrm{B}-\mathrm{C})$ 判断矩阵也是根据各影响因素的重要性进行的。 判断矩阵的最大特征值 $(\lambda$ max $)$ 和特征向量 $\omega=\left(\omega_{1} 、 \omega_{2}\right.$ 、 $\left.\omega_{3} 、 \cdots 、 \omega_{t}\right) T$ 为所求特征向量近似值, 及各因素权重。

表1 相对重要性标度。

\begin{tabular}{ll}
\hline 标度 & 定义 \\
\hline 1 & i因素与 $\mathrm{j}$ 因素相同重要 \\
3 & i因素比 $\mathrm{j}$ 因素略重要 \\
5 & i因素比 $\mathrm{j}$ 因素较重要 \\
7 & i因素比 $\mathrm{j}$ 因素非常重要 \\
9 & i因素比 $\mathrm{j}$ 因素绝对重要 \\
$2,4,6,8$ & 以上两判断之间的中间状态 \\
倒数 & i因素与 $\mathrm{j}$ 因素比较, 得到判断值为 $\mathrm{a}_{i j}=1 / \mathrm{a}_{j i}, \mathrm{a}_{i i}=1$ \\
\hline
\end{tabular}

第4步: 求同一层上的组合权系数。设当前层次上的 因素为 $\mathrm{A}_{1}, \cdots, \mathrm{A}_{n}$, 相关的上一层因素为 $\mathrm{C}_{1}, \cdots, \mathrm{C}_{m}$, 则对每个 $\mathrm{C}_{i}$, 根据第3步的讨论可求得一个权向量。按照 此方法按照层次结构图从上而下依次求下去, 一直到最后 一层所有影响阴损的权系数都计算出来为止, 按照每一层 各个影响因素的所占权重的分布就可以拟合出一个关于 不同方案优劣程度的简单排序。

第5步: 一致性检验。

一致性检验步骤为: 首先, 计算矩阵A的绝对一致性 指标 $\mathrm{CI}$, 由于矩阵中有众多因素需要进行两两比较, 这使 得判断矩阵的阶数 $\mathrm{n}$ 较大, 所以模型结果出现不一致性的 概率会变大, 因此还要对模型分析的结果进行随机一致性 $R I$ 检验以及相对一致性指标 $C R=C I / R I$ 的检验, 并且限定 $C R$ 时, 判断矩阵的一致性满意。

一致性指标:

$$
C I=\frac{\lambda \max -\mathrm{n}}{n-1}
$$

CI值愈大, 判断矩阵的完全一致性愈差, 一般只要求 CI不大于 0.1 , 认为判断矩阵的一致性可以接受, 否则必 须重新进行两两比较判别。对1 9阶矩阵, 平均随机一致 性指标RI见表 2 。 
表2 RI与维数的关系表。

\begin{tabular}{llllllllll}
\hline 阶数 & 1 & 2 & 3 & 4 & 5 & 6 & 7 & 8 & 9 \\
\hline RI & 0 & 0 & 0.58 & 0.90 & 1.12 & 1.24 & 1.32 & 1.41 & 1.45 \\
\hline
\end{tabular}

\section{4. 笁选影响因素构造互反矩阵}

\section{1. 篮选选址影响因素}

按照上文对层次分析法使用的基本要求, 现在进行大 型购物广场选址影响因素的精炼选取, 在整理归纳精炼的
过程中, 根据方元平、间小培, 、毕斗斗 (2007), 王怡文、 邵培基（2008），吴丽敏（2012）, 李依璘(2016)等人的 论文中提出的商业零售业企业选址的影响因素, 通过分析 购物广场的特点以及发展战略, 整合相似因素, 同时经过 企业管理专家的打分, 构造商业零售业选址影响因素, 并 且构造影响因素层次结构图, 如图1所示:

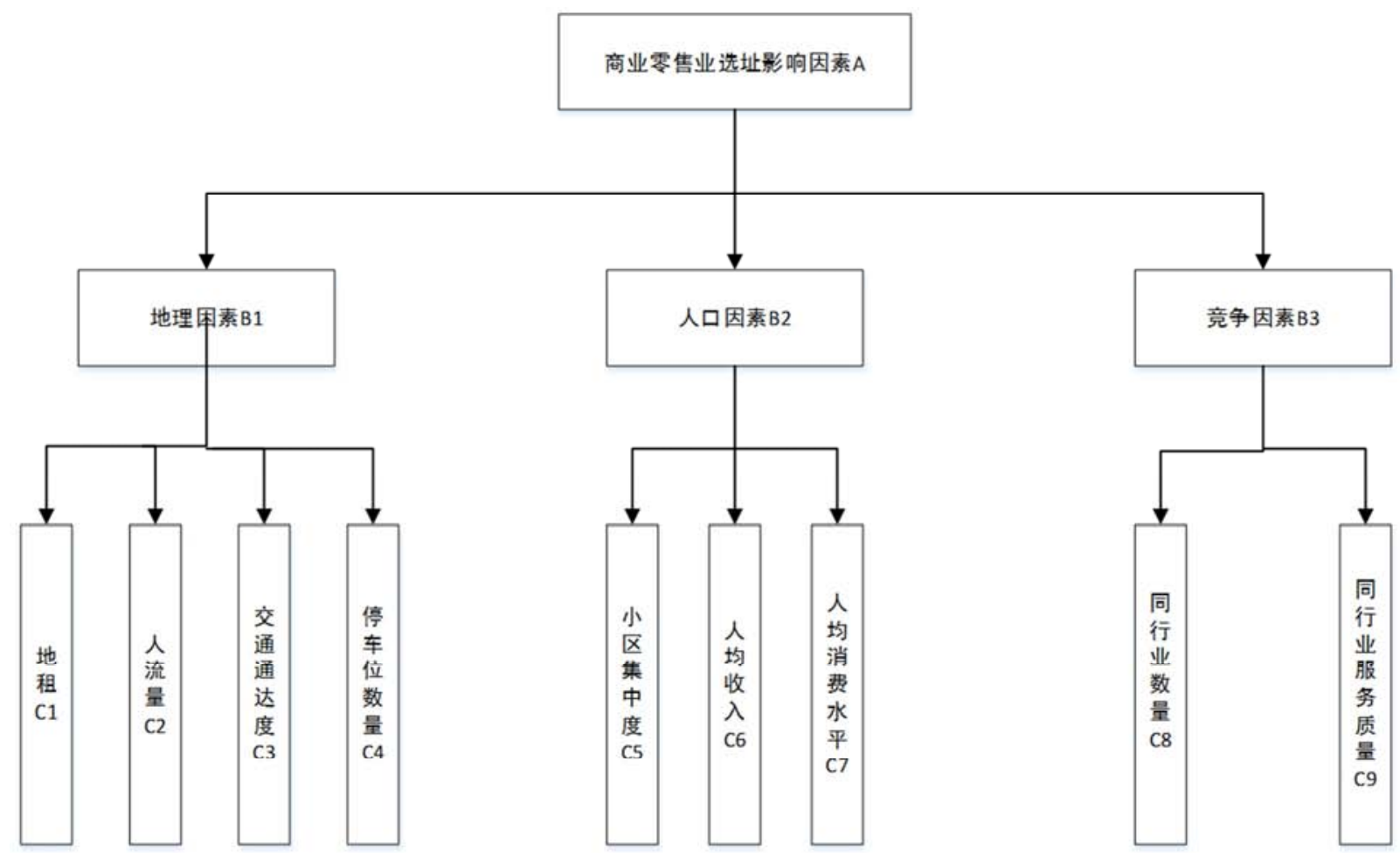

图1 商业零售业选址影响因素层次结构图。

商业零售业主要是提供消费和服务的场所, 地理位置 以及其服务的有效人口数量是十分重要的因素, 商业零售 业主要功能是服务消费者, 所以人口因素不可或缺。此外 商圈内现有的其他商业零售业的发展也是影响其选址的 重要因素。于是将商业零售业选址的影响因素细化为地理 因素B1、人口因素B2和竞争因素B3, 并将这三个因素作为 中间层因素。

在地理影响因素B1中, 主要是区位因素, 能够体现区 位因素的是地租 $\mathrm{C} 1$ 、人流量 $\mathrm{C} 2$ 、交通通达程度 $\mathrm{C} 3$ 和区位中 车位数量 $\mathrm{C} 4$ 。人口影响因素 $\mathrm{B} 2$ 中, 小区集中度 $\mathrm{C} 5$ 、人均收 入 $\mathrm{C} 6$ 和人均消费水平 $\mathrm{C} 7$ 占据支配地位。竞争影响因素 $\mathrm{B} 3$ 中, 主要体现在同行业之间竞争, 服务行业中影响同行业 竞争的因素主要是同行业数量 $\mathrm{C} 8$ 和同行业中其他企业提 供的服务质量C9。[7-10]

\section{2. 构造互反矩阵}

运用专家打分法并且依靠日常常识对地理因素B1, 人 口因素B2, 竞争因素B3构造互反矩阵。其中地理因素比人 口因素略重要 $\mathrm{a}_{12}=3$; 地理因素比竞争因素较重要 $\mathrm{a}_{13}=5$; 人口因素比竞争因素略重要 $\mathrm{a}_{23}=3$ 。互反矩阵 如下所示:

$$
\left(\begin{array}{ccc}
1 & 3 & 5 \\
1 / 3 & 1 & 3 \\
1 / 5 & 1 / 3 & 1
\end{array}\right)
$$

同理, 对地租因素 $\mathrm{C} 1$, 人流量因素 $\mathrm{C} 2$, 交通通达度因素 $\mathrm{C} 3$, 停车位数量因素 $\mathrm{C} 4$, 构造互反矩阵。 $\mathrm{a}_{12}=1 / 3 ; \mathrm{a}_{13}=3$; $\mathrm{a}_{14}=5 ; \mathrm{a}_{23}=5 \mathrm{a}_{24}=7 ; \mathrm{a}_{34}=3$ 。互反矩阵如下所示:

$$
\left(\begin{array}{cccc}
1 & 1 / 3 & 3 & 5 \\
3 & 1 & 3 & 7 \\
1 / 3 & 1 / 5 & 1 & 3 \\
1 / 5 & 1 / 7 & 1 / 3 & 1
\end{array}\right)
$$

对小区集中度因素 $\mathrm{C} 5$, 人均收入因素 $\mathrm{C} 6$, 人均消费水 平因素 $C 7$, 构造互反矩阵。 $\mathrm{a}_{12}=1 / 5 ; \mathrm{a}_{13}=1 / 3 ; \mathrm{a}_{23}=3$ 。 互反矩阵如下所示: 


$$
\left(\begin{array}{ccc}
1 & 1 / 5 & 1 / 3 \\
5 & 1 & 3 \\
3 & 1 / 3 & 1
\end{array}\right)
$$

对同行业数量因素C8和同行业服务质量因素C9构造 互反矩阵, 同行业质量因素比同行业服务质量因素略重要, $\mathrm{a}_{12}=3$ 。互反矩阵如下所示:

$$
\left(\begin{array}{cc}
1 & 3 \\
1 / 3 & 1
\end{array}\right)
$$

\section{3. 计算影响因素权重}

在得到商业零售业选址需求影响因素层次图并且用 1-9标度各个影响因素之间关系得到的互反矩阵后, 构造 判断矩阵, 下面就是运用 yaahp软件对上述因素构造判断 矩阵, 并且判断一致性比例, 具体如下表 3 到表6所示:

表3 对B1、B2、B3构造判断矩阵。

\begin{tabular}{lllll}
\hline A & B1 & B2 & B3 & Wi \\
\hline B1 & 1.0000 & 3.0000 & 5.0000 & 0.6333 \\
B2 & 0.3333 & 1.0000 & 3.0000 & 0.2605 \\
B3 & 0.2000 & 0.3333 & 1.0000 & 0.1062 \\
\hline
\end{tabular}

在上述表 3 中, 一致性比例为: 0.0372 ; 对 $A$ 的权重为 $1.0000 ; \lambda \max =3.0387$ 。一致性比例小于 0.1 , 所以能够 通过一致性检验。

表4 对 $\mathrm{C} 1 、 \mathrm{C} 2 、 \mathrm{C} 3 、 \mathrm{C} 4$ 构造判断矩阵。

\begin{tabular}{llllll}
\hline B1 & C1 & C2 & C3 & C4 & Wi \\
\hline C1 & 1.0000 & 0.3333 & 3.0000 & 5.0000 & 0.2633 \\
C2 & 3.0000 & 1.0000 & 5.0000 & 7.0000 & 0.5579 \\
C3 & 0.3333 & 0.2000 & 1.0000 & 3.0000 & 0.1219 \\
C4 & 0.2000 & 0.1429 & 0.3333 & 1.0000 & 0.0569 \\
\hline
\end{tabular}

在上述表 4 中, 一致性比例为: 0.0444 ; 对 $A$ 的权重为 $0.6333 ; \lambda \max =4.1185$ 。一致性比例小于 0.1 , 所以能够 通过一致性检验。

表5 对 C5、C6、C7构造判断矩阵。

\begin{tabular}{lllll}
\hline B2 & C5 & C6 & C7 & Wi \\
\hline C5 & 1.0000 & 0.2000 & 0.3333 & 0.1062 \\
C6 & 5.0000 & 1.0000 & 3.0000 & 0.6333 \\
C7 & 3.0000 & 0.3333 & 1.0000 & 0.2605 \\
\hline
\end{tabular}

在上述表5中,一致性比例为: 00372 ; 对 $A$ 的权重为 $0.2605 ; \lambda \max =3.0387$ 。一致性比例小于 0.1 , 所以能够 通过一致性检验。

表6 对 C8、C9构造判断矩阵。

\begin{tabular}{llll}
\hline B3 & C8 & C9 & Wi \\
\hline C8 & 1.0000 & 3.0000 & 0.7500 \\
C9 & 0.3333 & 1.0000 & 0.2500 \\
\hline
\end{tabular}

在上述表6中,一致性比例为: 0.0000 ; 对 $A$ 的权重为 $0.1062 ; \lambda \max =2.0000$ 。一致性比例小于 0.1 , 所以能够 通过一致性检验。
由四个判断矩阵计算得到的一致性比例均小于 0.1 , 则互反矩阵可以近似看做一致性矩阵, 整体可以通过了一 致性检验。可以将判断矩阵近似看做一致性矩阵, 再由一 致性矩阵运用yaahp软件进行各影响因素对商业零售业选 址的影响权重。

计算得各个影响因素在本层次中所占的权重:

表7 一级影响因素所占权重。

\begin{tabular}{llllll}
\hline 一级因素 & 权重 & 一级因素 & 权重 & 一级因素 & 权重 \\
\hline 地理因素 & 0.6333 & 人口因素 & 0.2605 & 竞争因素 & 0.1062 \\
\hline
\end{tabular}

表8 二级影响因素所占权重。

\begin{tabular}{llll}
\hline 二级因素 & 权重 & 二级因素 & 权重 \\
\hline 地租 & 0.1668 & 人均收入 & 0.1650 \\
人流量 & 0.3533 & 人均消费水平 & 0.0679 \\
交通通达度 & 0.0772 & 同行业数量 & 0.0796 \\
停车位数量 & 0.0360 & 同行业服务质量 & 0.0265 \\
小区集中度 & 0.0277 & & \\
\hline
\end{tabular}

一级影响因素中地理因素所占权重最大, 为 0.6333 , 其次是人口因素所占权重为 0.2605 , 占比重最少的是竞争 性因素, 为 0.1062 。地理因素对购物广场选址有很大影响, 在选址过程中要首先考虑地理因素, 按着每种因素影响力 其次考虑人口因素的影响, 再考虑竞争因素对选址的影响。

二级影响因素中人流量因素最为重要, 占 0.3533 , 商 业零售业人流量是客流量的直接反映。时人均收入因素和 地租因素相对重要, 人均收入水平直接影响消费者购买力, 不同的收入水平消费观念和消费水平都存在很大差距; 地 租因素是销售成本中重要的组成部分, 也是商业区位重要 组成要素。其次, 同行业数量以及交通通达度等因素对商 业零售业的选址也有一定影响, 同行数量在形成同行业的 集聚效应的同时，作为销售服务行业也会形成相当大的竞 争, 在竞争力相差水平不大情况下会分散消费者, 导致经 营效应下降。人均消费水平也有一定影响, 占总权重的 0.0679 , 人均消费水平在一定程度上反映了该地区消费者 的消费习惯和消费观念, 这也在一定程度上影响了商业零 售业的选址。最后, 小区集中度和同行业服务质量以及停 车位数量这三个因素对商业零售业选址影响较小, 原因可 能是现在商业区都很少保留车位而且交通比较发达, 大部 分小区由于地价原因也都不集中在商业区周边, 规模相当 的两个竞争企业在给消费者提供的服务商也不会相差很 大, 基本属于同质类型, 所以说这三个影响因素在影响商 业零售业选址方面所占权重比例比较低。

因此, 在商业零售业选址的过程中, 在充分考虑各种 影响因素的前提条件下要按照各种影响因素所占权重来 考虑各个因素的优先性, 也可以根据各个影响因素所占权 重给给定的多个选址地点进行打分, 最后来确定最后选址 地点。

如何从多个备选方案中选出最适宜的选址方案就涉 及到了选址适宜性综合评价 [11]。可采用采用多目标决策 现行加权方法来确定, 建立一个目标函数确定每种方案综 合评分:

$$
\mathrm{Z}=\sum_{\mathrm{i}=1}^{\mathrm{n}} Z_{\mathrm{i}}
$$


上式 (2) 中: $Z$ 为某备选地址适宜性总评分; $i$ 为第 一层制约因素第 $i$ 项影响因素; $n$ 为此备选地址第一层制约 因素的个数; $Z_{i}$ 为第一层制约因素第 $\mathrm{i}$ 项影响因素的总分。

$$
\mathrm{Z}_{\mathrm{i}}=\sum_{L=1}^{\mathrm{k} 1} K_{\mathrm{i} 00} K_{\mathrm{ij0}} K_{\mathrm{ij} L} K_{\mathrm{ij} L S}
$$

上式 (3) 中, $\mathrm{Z}_{i}$ 位第一层制约因素第 $\mathrm{i}$ 项影响因素的 总分; $i$ 为第一层制约因素个数; $j$ 为第一层制约因素第 $i$ 项影响因素的第二层因素 $j$ 自因素, $j=0,1,2$, ; L位第二层 制约因素第 $\mathrm{j}$ 项影响移速第三层子因素的第 $\mathrm{L}$ 子因素, $\mathrm{L}=0,1,2, \cdots \cdots, \mathrm{n} ; \mathrm{K} 1$ 位第一层制约因素 $\mathrm{i}$ 项影响因素的个 数, $\mathrm{K} 1=0,1,2, \cdots \cdots, \mathrm{n} ; K_{i 00}$ 为第一层制约因素第 $\mathrm{i}$ 子因素 权重; $K_{i j 0}$ 为第二层制约因素第 $\mathrm{j}$ 因素权重; $K_{i j L}$ 为第三层 制约因素第L因素权重; $K_{i j L S}$ 为第三层制约因素第L因素实 际贡献权重。若按百分制计算, 层次分析综合评价模型为:

$$
\mathrm{Z}=\mathrm{Z}_{i}=100 \sum_{i=1}^{n} \sum_{L=1}^{k 1} K_{i 00} K_{i j 0} K_{i j L} K_{i j L S}
$$
案。

最后, 根据备选地址的得分情况选择最适宜的选址方

\section{5. 研究不足之处}

运用层次分析法在进行选址的过程中存在以下几点 不足之处:

首先，不能生成新方案。运用层次分析法进行选址只 能在给定备选方案的基础上进行选择最优者, 不能在原有 的基础上提供更好的可行位置。由于只能从备选方案中进 行选择这就得使用此方法进行区位选择缺乏创新性。[12]

第二, 层次分析法定量数据较少, 定性成分多。特别 是运用专家评分法对同一层次不同因素进行两两比较时, 人为定性, 完全依靠主观经验进行打分, 与严格的数学论 证和完善的定量方法有很大不同, 说服力较低。[13]

第三, 当指标过多时, 要素权重往往难以确定。在解 决本文商业零售业选址问题上选取影响因素数量适中, 调 理清楚。但是, 扩展到较普遍的问题时, 随着指标体系变 大, 选取因素变多时要确定的关系就非常多, 容易出现错 误。

\section{6. 结论}

在研究选址影响因素过程中, 本文运用层次分析法从 新视角进行分析研究。尽管许多国外学者已经从多角度对 商业区位选择进行了细致的研究, 但是国内研究仍然对商
业布局、连锁超市、购物中心等零售服务业关注不足。当 然, 运用层次分析法对商业零售业选址进行研究分析时也 存在上述不足, 可能还需要继续研究发展, 与更多的研究 方法相结合。

\section{参考文献}

[1] 李依璘. 基于 GIS 的零售业选址问题分析 [J]. 商业经济研 究, 2016, (07) :22-23。

[2] 钟太勇, 靳强. 基于层次分析法的连锁店选址研究——以十 堰市某连锁超市为例 $[\mathrm{J}]$. 郧阳师范高等专科学校学 报, 2014,(03):27-30。

[3] Neil Carn, Joseph Rabianski, Ronald Racster etc. "Real Estate Market Analysis: Techniques and Applications” CITIC Publishing House, 2005.

[4] 方远平, 间小培, 毕斗斗. 1980年以来我国城市商业区位研 究述评 [J]. 热带地理, 2007, (05) : 435-440。

[5] 陈焕娣. 浅谈模糊综合评价法在家电连锁零售企业门店选 址中的应用 $[\mathrm{J}]$. 科技信息, 2009, 33:514-515。

[6] 邓雪, 李家铭, 曾浩健, 陈俊羊, 赵俊峰. 层次分析法权重计 算方法分析及其应用研究 $[\mathrm{J}]$. 数学的实践与认 识, 2012, (07):93-100。

[7] 方远平, 间小培, 毕斗斗. 1980年以来我国城市商业区位研 究述评. 热带地理, 2007, 27 (5) : 435-440。

[8］王怡文, 邵培基. 基于企业与顾客双重视角的连锁型服务企 业选址影响因素研究 $[\mathrm{J}]$. 管理学家 (学术 版), 2008, 06:547-552+559。

[9] 吴丽敏. 基于顾客满意度的配送中心选址研究 [D]. 广东商 学院, 2012。

[10] 李依璘. 基于 GIS 的零售业选址问题分析 [J]. 商业经济研 究, 2016, (07) :22-23。

[11] 钱光毅. 填埋场选址的理论方法及实际应用 $[\mathrm{J}]$. 四川建筑 科学研究, 2010, (01) :255-257。

[12] 汪玉萍. 全国各省市影响企业选址的因素分析一一基于因 子分析和聚类分析法 [J]. 现代商业, 2014, 33:280-281。

[13] 王士君, 浩飞龙, 姜丽丽. 长春市大型商业网点的区位特征 及其影响因素 $[J]$. 地理学报, 2015, 06:893-905。 